\title{
Immunological Response of Hiv-Infected Children to Highly Active Antiretoviral Therapy at Gondar University Hospital, North-Western Ethiopia
}

\author{
Mehretie Kokeb ${ }^{1}$, Getu Degu ${ }^{2}$
}

\begin{abstract}
BACKGROUND: The effectiveness of highly active antiretroviral therapy (HAART) in children has not been well studied specially in developing countries where the burden of HIV is high. This study was aimed to assess the immunologic response of HIV-infected children to HAART at Pediatric ART Clinic Gondar University Hospital.

METHODS: Institution based cross-sectional study was conducted at the Pediatric ART Clinic Gondar University Hospital from March01-April 30, 2014. The study included $283 \mathrm{HIV}$-infected children who were on HAART for 6 months and above. Medical records of HIV-infected children were reviewed using pre-tested questionnaire. CD4 count/percent was collected every 6 months retrospectively. For all statistical significance tests, the cut-off value was $p<0.05$. Poison Regression was used for further analysis.

RESULTS: The mean age of children was 6.9 years with a standard deviation of 3.4 years. The median CD4 count/percent was 232/13\%, 450/21\%, 540/25\% and 608/27\% at the time of initiation, 6, 12 and 18 months of ART, respectively. HAART initiated at higher CD4 count, good adherence and HIV status disclosure were found to have positive effects for immunological response.

CONCLUSION: The study revealed that there was good Immunological response to ART, and that the maximum response was in the $1^{\text {st }} 6$ months of ART. Low CD4 count at initiation, undisclosed HIV status and lack of good adherence were found to cause low immunological response to HAART.
\end{abstract}

KEYWORDS: HAART, CD4, Immunologic response

DOI:http://dx.doi.org/10.4314/ejhs.v26i1.6

\section{INTRODUCTION}

About 2.1 million children of less than 15 years old are living with HIV worldwide. Of these, $90 \%$ live in sub-Saharan Africa and about 135,813 live in Ethiopia (1). ART has radically changed the natural course of HIV infection in countries where it has been successfully implemented and HIVinfected infants and children now survive to adolescence and adulthood (2). In resource-limited countries, despite many challenges to program implementation, committed efforts have resulted in remarkable progress in making ART accessible to HIV-infected children. Significant challenges to the scale-up of pediatric care and treatment include: limited screening of HIV-infected children, lack of affordable and simple diagnostic tests, lack of trained human resources, insufficient advocacy and lack of understanding that ART is efficacious in children $(3,4)$. WHO recommended that first line ART drugs are having good result in increasing the CD4 count and suppressing the plasma viral load in children in resourceconstrainedregions,. Making the ART drugs free of charge is related to good outcome of children $(5,6,7)$. Clinical response with increment in anthropometric parameters, lowering of WHO clinical stages and reduction in incidence of opportunistic infections were achieved after

${ }^{1}$ Department of Pediatrics and Child Health, University of Gondar, Ethiopia

${ }^{2}$ Department of Epidemiology and Biostastics, University of Gondar, Ethiopia

Corresponding Author: Mehretie Kokeb, Email: mehretiek@gmail.com 
HAART in children $(8,9,10)$. The rationale for conducting this research was the fact that there is no adequate study done on immunologic response of HIV-infected children in Ethiopia, and no study has been done at Gondar University Hospital In addition, the number of children being started on HAART is increasing in Ethiopia, which necessitates clear knowledge about the treatment response. The major objective of our study was to assess the immunologic response of HIV-infected children to Highly Active Antiretroviral Therapy focusing on the periodic evaluation of $\mathrm{CD} 4$ count/percent.

\section{METHOD AND MATERIALS}

An institution based cross-sectional study was conducted at the Pediatric ART Clinic, Gondar University Hospital, from March-April 2014 to assess the immunologic response of HIV-infected children to HAART. The medical records of all HIV-infected children having followup at Gondar University Hospital, Pediatric ART Clinic, and took HAART for 6 months or more were reviewed. Sample size was calculated to be 340 which was obtained by systematic random sampling technique taking every $4^{\text {th }}$ record number from a total of $1360 \mathrm{HIV}$-infected children taking HAART for 6 months or more. HIV-infected children with no documented $\mathrm{CD} 4$ results at initiation and/or 6 months were excluded from the study. Out of 340 medical records, 57 were excluded from analysis because of lack of documented CD4 results at initiation (30 cases) and at 6 months of HAART (27cases). Medical records of the sampled population were collected by two clinical nurses working at the ART Clinic (one working at Pediatric ART, one working at Adult ART) under the supervision of one physician. The collected patient records were reviewed based on the required variables: age, WHO clinical stage (1-4) at initiation and CD4 count/percent at initiation, adherence, relation with the care taker, parental HIV status, disclosure status, and survival status of mother. The questionnaire was pretested and structured; the pretest was done on 25 patients $(8 \%$ of the sample) who were not included in the study. All data collected for the study were double entered in SPSS by two different data entry clerks and verified using the validation program. In the analysis, the socio-economic and demographic data were related with the immunological results of HIV-infected children taking HAART. For all statistical significance tests, the cut-off value was $\mathrm{p}<0.05$. Poison Regression was used for further analysis of the outcome of HIV-infected children. The problem of extra-poison variation was checked using the usual assessment mechanism, and there was no over dispersion as the deviance (i.e. $-2 \log$ likelihood) divided by the corresponding degree of freedom was very close to 1. Ethical approval for this study was secured from internal review board at the University of Gondar, College of Medicine and Health Sciences. Permission letter to conduct the research was obtained from Gondar University Hospital. Confidentiality was secured by coding each medical record without mentioning the name of the patient.

\section{RESULTS}

A total of $283 \mathrm{HIV}$-infected children who were on ART for 6 months or more were included in the study. Of these, $146(51.6 \%)$ were males while the remaining 137 (48.4\%) were females. Most of the cases were from Gondar Town which consisted of $75 \%(212 / 283)$. The remaining $25 \%$ were out of the town of Gondar (Table 1).The age distribution showed that $185(65.4 \%), 85(30 \%)$, and $13(4.6$ $\%$ ) children were 5-14yrs, 1-5yrs and below 1 year, respectively. The Minimum age at initiation was 6 months and the maximum was 17years with a median of 6.9 years and a standard deviation of 3.4 years. The study showed that $31.1 \%(88 / 283)$ of mothers had unknown HIV status and the remaining $68.9 \%$ were confirmed HIV- positive; $140(49.5 \%), 119(42 \%)$, and $24(8.5 \%)$ fathers were HIV-positive, unknown status and negative, respectively (Table-1). The majority of children were tested for their HIV-infection status because of illness consisting of $213(75.3 \%)$ and the rest $40(14.1 \%), 10(3.5 \%)$ and $20(7.1 \%)$ were tested because parent/s positive for HIV, for VCT and Provider-initiated Testing and counseling, respectively. In this study, 57(20.2 \%), $135(47.7 \%), 65(22.9 \%)$ and $26(9.2 \%)$ of the children were at clinical stages $4,3,2$ and 1 , respectively at initiation of ART.Clinical Stage 1 was observed in 149(53.9 \%) at 6 months, $147(55.5 \%)$ at 12 months and $231(78 \%)$ at 18 months of HAART (Figure 1).The median CD4 count and CD 4 percent at initiation were 232 and 
$13 \%$, respectively with a maximum CD4 count of 2300 and a maximum CD4 percent of $96 \%$ and the minimum CD4 count and CD4 percent were 2 and $0.15 \%$, respectively. The median CD4 count and percent were $450 / 21 \%, 540 / 25 \%$ and $608 / 27 \%$ at 6 months, 12 months and 18 months of HAART, respectively (Table 2 ).

Table1: Socio-demographic characteristics of the study population from march 01-April 30, 2014 at Pediatric ART Clinic, Gondar University Hospital.

\begin{tabular}{|c|c|c|}
\hline \multicolumn{3}{|c|}{ Socio-demographic Characteristics } \\
\hline Variables & Number $(n=283)$ & Percent $(\%)$ \\
\hline \multicolumn{3}{|l|}{ Age (Years) } \\
\hline$<1$ & 13 & 4.6 \\
\hline $1-3$ & 43 & 15.2 \\
\hline $3-5$ & 42 & 14.8 \\
\hline$>5$ & 185 & 65.4 \\
\hline \multicolumn{3}{|l|}{ Maternal HIV Status } \\
\hline Positive & 195 & 68.9 \\
\hline Unknown & 88 & 31.1 \\
\hline \multicolumn{3}{|l|}{ HIV status of Father } \\
\hline Positive & 119 & 42 \\
\hline Negative & 24 & 8.5 \\
\hline Unknown & 140 & 49.5 \\
\hline \multicolumn{3}{|c|}{ Educational status of Mother } \\
\hline Could not read / write & 110 & 38.9 \\
\hline Read and Write & 68 & 24 \\
\hline Elementary & 72 & 25.4 \\
\hline Secondary & 26 & 9.2 \\
\hline Tertiary & 7 & 2.5 \\
\hline \multicolumn{3}{|l|}{ Educational Status of Father } \\
\hline Could not read/write & 101 & 35.7 \\
\hline Read and Write & 56 & 19.8 \\
\hline Elementary & 78 & 27.6 \\
\hline Secondary & 25 & 8.8 \\
\hline Tertiary & 23 & 8.1 \\
\hline
\end{tabular}

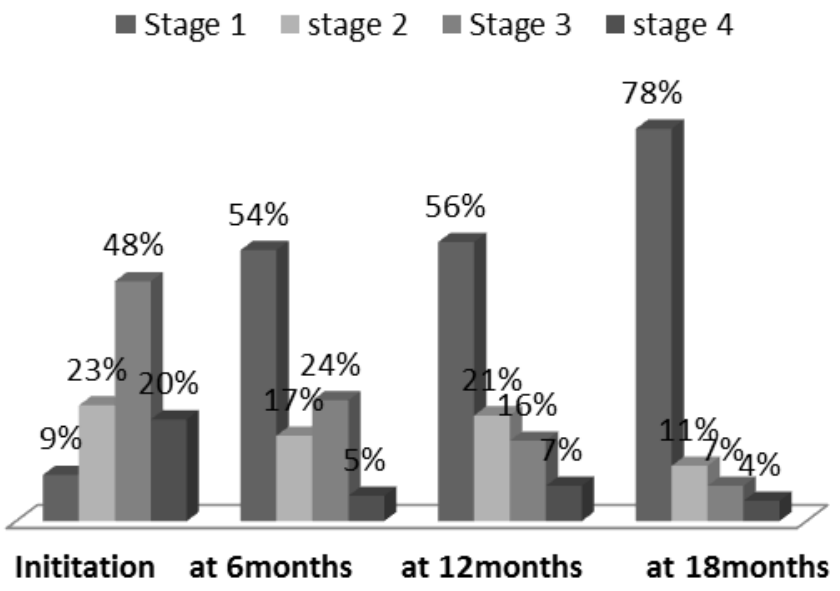

Figure 1: Clinical stages at initiation, 6 months, 12 months and 18 months of ART, GUH 
Table 2: Median CD 4 count/percent at 0, 6, 12 and 18 months of ART

\begin{tabular}{|c|c|c|c|c|}
\hline \multicolumn{5}{|c|}{ Months on HAART } \\
\hline Median & 0 (at initiation) & 6 & 12 & 18 \\
\hline CD4 count & 232(Max:2300, Min:2) & $\begin{array}{c}\text { 450(Max:3338, } \\
\text { Min:12) }\end{array}$ & $\begin{array}{c}\text { 540(Max:2000, } \\
\text { Min:14) }\end{array}$ & $\begin{array}{c}\text { 608(Max:2000, } \\
\text { Min:16) }\end{array}$ \\
\hline CD4 percent & 13(Max: 96, Min: $0.15 \%$ ) & $\begin{array}{c}21(\operatorname{Max}: \quad 89, \\
\text { Min: } 0.2 \%)\end{array}$ & 25(Max:94, Min: $3 \%$ ) & $\begin{array}{c}\text { 27(Max:99, Min: } \\
10 \%)\end{array}$ \\
\hline
\end{tabular}

In this study, 6(2.1\%), 9(3.3\%) and $1(0.4 \%)$ children died at 6,12 and 18 months of HAART, respectively, making a total of $16(5.7 \%)$ deaths during the 18 months followup. Disclosure of HIV status was given to $20(7.1 \%), 29(10.5 \%)$, $34(12.8 \%)$, and $43(16.4 \%)$ of children at the initiation, 6, 12 and 18 months of HAART, respectively (Table 3). Adherence was found to be good during the whole 18 months period and $95.5 \%, 97.8 \%$ and $97.6 \%$ of children at 6,12 and 18 moths of HAART were reported to have good adherence, respectively, according to the Ethiopian Pediatric ART guideline 2008. The median CD4 percent at 6 months of HART was found to be $24.1 \%, 20.1 \%$ and $18.1 \%$ when initiated in children of age below 3years, 3-5years and above 5years, respectively. Median CD4 percent at 6 months of HAART was $16.4 \%, 24.6 \%$ and $34 \%$ when treatment was initiated at CD4 count of less than 200, 200-500 and more than or equal to 500, respectively. Median CD4 percent at 6 months of treatment was $20.2 \%, 10.7 \%$ and $9.2 \%$ in children with Good, Fair and Poor adherence, respectively. The median CD4 percent at 6 moths of HAART was found to be $20.8 \%, 24.8 \%, 12.6 \%$ and $8.1 \%$ in children with clinical stage of I, II, III \& IV at initiation, respectively (Table 4).

Initial CD4 count, disclosure of HIV status and adherence were found to have statistically significant associations with $\mathrm{CD} 4$ response at 6 months of HAART. In this regard, children with higher initial CD4 count were found to have good increment of CD4 at 6 months of HAART. Children whose disclosure was given at initiation of HAART were observed to have about $34 \%$ increase in CD4 count compared to those who did not get their status disclosed at 6 months at

Table 4: Median CD4 percent at 6 months of HAART in relation to the independent variables.

\begin{tabular}{ll}
\hline Variable & $\begin{array}{l}\text { Median CD4 } \\
\text { percent after } \\
\text { 6months of ART }\end{array}$ \\
\hline Age & \\
$\quad$ Less than 3years & 24.1 \\
3-5years & 20.1 \\
> 5years & 18.4 \\
CD4 count at initiation & \\
$\quad$ Less than 200 & 16.4 \\
200-500 & 24.6 \\
>500 & 34 \\
Adherence & \\
Good & 20.2 \\
Fair & 10.7 \\
Poor & 9.2 \\
Clinical stage at initiation & \\
I & 20.8 \\
II & 24.8 \\
III & 12.6 \\
IV & 8.1 \\
\hline
\end{tabular}

HAART. On the other hand, those with poor adherence were found to have a $16 \%$ lower CD4 increment compared to those with good adherence. Age showed a marginal significance while child's sex, maternal death, and clinical stage did not show a statistically significant association with the outcome variable (Table 5).

Table 3: Disclosure at initiation, 6, 12 and 18 months of HAART

\begin{tabular}{lllll}
\hline \multicolumn{5}{c}{ Months on HAART } \\
\hline HIV status & Initiation & 6 & 12 & 18 \\
\hline Not Disclosed & $263(92.9 \%)$ & $247(89.5 \%)$ & $231(87.2 \%)$ & $220(83.6 \%)$ \\
Disclosed & $20(7.1 \%)$ & $29(10.5 \%)$ & $34(12.8 \%)$ & $43(16.4 \%)$ \\
\hline
\end{tabular}


Table 5: Effect of selected independent variables using Poison Regression with CD4 count at 6 months of HAART.

\begin{tabular}{llllll}
\hline Variable & Coefficient (b) & $\begin{array}{l}\text { Relative rate } \\
\text { (rate ratio) }\end{array}$ & $95 \%$ C.I. & & P-value \\
\hline Initial CD4 count & 0.0099 & 1.01 & 1.001 & 1.019 & $<0.001^{*}$ \\
Age & -.0222 & 0.978 & 0.955 & 1.003 & 0.081 \\
Sex & -.0377 & 0.963 & 0.825 & 1.125 & 0.636 \\
Mother alive & -.1031 & 0.902 & 0.764 & 1.066 & 0.226 \\
Disclosure & 0.2919 & 1.339 & 1.073 & 1.672 & $0.01^{*}$ \\
Adherence & -.1732 & 0.841 & 0.730 & 0.969 & $0.017^{*}$ \\
Clinical stage 1 & 0.0315 & 1.032 & 0.794 & 1.34 & 0.816 \\
Clinical stage 2 & -.1087 & 0.897 & 0.747 & 1.078 & 0.247 \\
Clinical stage 3/4 & -.0336 & 0.967 & 0.805 & 1.161 & 0.717 \\
\hline
\end{tabular}

\section{DISCUSSION}

The primary aim of this study was to assess the immunological response of $\mathrm{HIV}$-infected children to HAART after 6 months of treatment. Our study showed that the majority of children $(75.3 \%)$ were diagnosed for HIV because they were sick and only few (7.1\%) were diagnosed by Provider-Initiated Counseling and Testing. This shows that children are diagnosed after they get deteriorated which may be a reason for poor response to HAART. Thus, there should be strong PITC activity to catch children before advanced HIV disease. The majority $(65.4 \%)$ of the children were diagnosed for HIV after 5 years of age with the median age of 6.9 years, which is lower than a study in Kenya which revealed mean age of 8.5 years (1) but significantly higher than a study in USA where the median age at initiation was 2.7 years (2). This shows that initiation of HAART in the study area is not being done in early age which is a very important factor for response to HAART. This study revealed that the majority of the children $(67.9 \%)$ were started on HAART at severe clinical stage (Stage 3 or 4) which is almost comparable with a study from South Africa which revealed $62.7 \%$ were in severe clinical stage at initiation (3) but higher thana study from India which revealed only $40.3 \%$ were at severe stage when they were initiated(4). This indicates that children are not being started on HAART earlier which may be due to late diagnosis of HIV in children. The median CD4 count/CD4 percent at initiation was 232(13\%), with maximum CD4 count/CD4 percent of $2300 / 23 \%$, and the minimum CD4 count/ CD4 percent was $2 / 0.15 \%$. The median CD4 counts/percents were 450/21\%, 540/25\% and $608 / 27 \%$ at 6 months, 12 months and 18 months of ART, respectively. Significant increment was shown during the first 6 months of therapy (from 232/13\% to $450 / 21 \%$ ).

The response rate at 6 months is almost similar with the analysis done in South Africa; a similar finding was also reported from Thailand $(3,9)$. This finding is in consistent with other studies showing a significant raise during the first 6 months of $\operatorname{HAART}(6,7,8)$. This is good evidence that children are responding well to HAART, and in this study, the CD4 at 6 months was higher compared to the same analysis in Kenya which revealed median of 293 at 6 months of HAART (6). Immunological response was found to be better in children less than 5 years compared to those more than 5 years at initiation of HAART (median CD4 percent at 6 months was $24.1 \%$ vs $18.4 \%$ ). This is comparable with the European collaborative study which revealed that CD4 response was significantly higher when HAART is started before 5 moths of age (10). This is evidence that children should be started on treatment in early age to have good immunological response which is most likely related to immunological deterioration if not treated earlier. The CD4 percent at 6 months of HAART was found to be significantly higher in those children who started therapy at CD4 count of more than 500 in comparison to those started below $200 \quad(34 \%$ vs $16.4 \%)$. This shows that even if HAART is given, the response will be poor if started after the immunity is significantly damaged. Children who started therapy at clinical stage 4 were found to have very low response after 6 months compared to children who started at clinical stage $1(8.1 \%$ vs $20.8 \%)$. This is shows that starting treatment after severe manifestation will not bring ssignificant change in CD4 response because of the already deteriorated immunity. Therefore, starting HAART before clinical and/or immunological deterioration is important to get good CD4 response with the treatment. Similar findings were revealed in different studies $(11,12$, and 13).

Compared with other predictors, our analysis revealed that initiation of HAART at low CD4 count $(\mathrm{p}<0.001)$, children with no good adherence and not disclosed of their HIV status were having significant effect on CD4 count increment at 6months of HAART 
$(\mathrm{P}<0.05)$. It was also observed that age had shown a borderline effect on immunologic response ( $\mathrm{p}$-value of $0.081), 95 \%$ CI $(0.955,1.003)$. According to this finding (although not significant at the usual 5\% level of significance), initiation of HAART at a relatively older age was followed by a relatively reduced (2\%) CD4 count on the average. In other words initiation of HAART at early age seemed to improve the immunologic response. This finding is in complete agreement with the result of a study undertaken in Jimma, Ethiopia, which showed strong evidence that early initiation has a good effect on immunologic response (14). This finding is a good reason to have diagnostic tests at early age which is one of the major challenges in resource-limited areas due to the need to have sophisticated tests like Polymerase Chain Reaction.

\section{ACKNOWLEDGMENTS}

We are grateful to the University of Gondar for sponsoring this research. We also extend our gratitude to data collectors specially Mrs.Yayesh Tassew and Sr.Helen Sibhat. In addition, we acknowledge Gondar University Hospital for allowing us to use patient documents.

\section{REFERENCES}

1. Rinn Song, Justine Jelagat, Doris Dzombo, Marietta Mwalimu, Kishorchandra Mandaliya, Khadija Shikely et.al. Efficacy of Highly Active Antiretroviral Therapy in HIV-1 Infected Children in Kenya. PEDIATRICS , 2007; 4:10-15.

2. Watson, Douglas C. MD; Farley, John J. MD, MPH. Efficacy of and adherence to highly active antiretroviral therapy in children infected with human immunodeficiency virus type 1. The Pediatric Infectious Disease Journal, 1999; 8(18):682-689.

3. Anand Reddi, Sarah C Leeper, Anneke C Groble. Preliminary outcomes of a pediatric highly active antiretroviral therapy cohort from KwaZulu-Natal, South Africa. BMC Pediatrics, 2007; 7: 13 doi:10.1186/1471-2431-7-13.

4. N. Kumarasamy, Kartik K. Venkatesh, Bella Devaleenol, S. Poongulali, S. N. Mothi and Suniti Solomon.Safety, Tolerability and Effectiveness of Generic HAART in HIV-Infected Children inSouth India. Journal of Tropical Pediatrics, 2009; 55(3):155-159.

5. Parakh A, Dubey AP, Kumar A, Maheshwari A, Saxena R. Efficacy of first-line, WHO recommended generic HAART regimens in Indian children. Kathmandu University Medical Journal, 2009; 7: 220-225.
6. Dalton C. Early Response to Highly Active Antiretroviral Therapy in HIV-1-Infected Kenyan Children. J Acquir Immune Defic Syndr, July 2007; 3.

7. D Germanaud, A Derache, $M$ Traore, $Y$ Madec, S Toure, F Dicko, H Coulibaly, M Sylla, V Calvez and AG Marcelin.Virological response to first-line HAART in HIV-1-infected children in Mali: Rate of failure and Resistance after 6months of ART. Antivir Ther, 2009; $14: 150-154$

8. Philippa M Musoke, Peter Mudiope, Linda N Barlow-Mosha. Growth, immune and viral responses in HIV infected African children receiving highly active antiretroviral therapy: a prospective cohort study. BMC Pediatrics, 2010; 10:56.

9. Thanyawee Puthanakit, Aurmporn Oberdorfer, Noppadon Akarathum, Suparat Kanjanavanit, Pornphun Wannarit, Thira Sirisanthana and Virat Sirisanthana. Efficacy of Highly Active Antiretroviral Therapy in HIV-Infected Children Participating in Thailand's National Access to Antiretroviral Program.CID, 2005;41:1.

10. Michael Louie, Christine Hogan, Michele Di Mascio,et.al. Determining the Relative Efficacy of Highly Active Antiretroviral Therapy. The Journal of Infectious Diseases, 2003; 187:896-900.

11. Ann J. Melvin, MD, MPH; Paul F. Lewis, MD; Kathleen M. Mohan, RN, MS; W. Scott Naugler, MD; Lisa M. Frenkel, MD.Efficacy and Toxicity of Antiretroviral Therapy Using 4 or More Agents Application of a Strategy for Antiretroviral Management in Human Immunodeficiency VirusInfected Children. Arch Pediatr Adolesc Med, 2002; 156:568-573.

12. Daniel Glikman, MD, Linda Walsh, NP, Judy Valkenburg, PA-C, P. Daisy Mangat, RN, MPH, John F. Marcinak, MD. Hospital-Based Directly Observed Therapy for HIV-Infected Children and Adolescents to Assess Adherence to Antiretroviral Medications. PEDIATRICS, May 2007; 119:5.

13. Jensen-Fangel $\mathrm{S}$. The effectiveness of highly active antiretroviral therapy in HIV-infected patients. Dan Med Bull, 2004; 51:371-92.

14. Netsanet Workneh, MD, Tsinuel Girma, MD, Mirkuzie Woldie, MD, MPH. Immunologic and clinical outcomes of children on HAART: a retrospective cohort analysis at Jimma University specialized hospital. Ethiop J Health Sci, 2009;19: 2. 